\title{
Effects of Stepwise Lung Recruitment Maneuvers in Patients with Early Acute Respiratory Distress Syndrome: A Prospective, Randomized, Controlled Trial
}

\author{
Shu-Chen Kung ${ }^{1,+}{ }^{,}$Yi-Li Hung ${ }^{2,3,+}$, Wan-Ling Chen ${ }^{1}$, Ching-Min Wang ${ }^{4}$, Hui-Chun Chang ${ }^{1}$ \\ and Wei-Lun Liu ${ }^{3,5, *(D)}$ \\ 1 Department of Respiratory Therapy, Chi Mei Medical Center, Liouying, Tainan 73657, Taiwan; \\ clh7810@mail.chimei.org.tw (S.-C.K.); wanlin0810@gmail.com (W.-L.C.); smallwa999@gmail.com (H.-C.C.) \\ 2 Department of Pediatrics, Cathay General Hospital, Taipei, Taiwan; b82401103@yahoo.com.tw \\ 3 School of Medicine, College of Medicine, Fu Jen Catholic University, Xinzhuang Dist., \\ New Taipei City 24205, Taiwan \\ 4 Department of Internal Medicine, Chi Mei Medical Center, Liouying, Tainan 73657, Taiwan; \\ wangchingmin@yahoo.com.tw \\ 5 Division of Critical Care Medicine, Department of Emergency and Critical Care Medicine, \\ Fu Jen Catholic University Hospital, Fu Jen Catholic University, New Taipei City 24352, Taiwan \\ * Correspondence: medrpeterliu@gmail.com; Tel.: +886-2-8512-8888 (ext. 22200); Fax: +886-2-85128771 \\ + Both authors contributed equally to this work.
}

Received: 23 January 2019; Accepted: 6 February 2019; Published: 10 February 2019

\begin{abstract}
Since the clinical benefit of lung recruitment maneuvers (LRMs) is still conflicting, we performed this prospective, randomized, controlled study to investigate whether LRMs should be used in the routine management of acute respiratory distress syndrome (ARDS). This trial was conducted in four intensive care units (ICUs) to compare application of a modified stepwise LRMs with solely lung-protective ventilation in patients with moderate to severe ARDS within $72 \mathrm{~h}$ from the onset. The primary outcome was 28-day mortality, and the secondary outcomes were ventilator-free days and ICU-free days. We collected data on 120 ARDS patients from 2009 to 2012, and there was no difference in 28 -day mortality between the two groups ( $28.3 \%$ vs. $30.0 \%, p=0.84)$. However, among survivors, patients in the LRM group had a significant longer median duration of ventilator-free days (18 vs. 13 days; $p=0.04)$ and ICU-free days ( 16 vs. 11 days; $p=0.03$ ) at 28 days than in the control group. The respiratory system compliance was significantly higher in the LRM group from day 1 to day 7. The occurrence rate of barotrauma was similar in both groups. We concluded that LRMs combined with lung-protective ventilation in early ARDS may improve patient outcomes.
\end{abstract}

Keywords: acute respiratory distress syndrome; stepwise lung recruitment maneuver; ventilator-free days; ICU-free days

\section{Introduction}

Acute respiratory distress syndrome (ARDS) is a hypoxic, non-homogeneous pulmonary disease characterized by focal atelectasis, focal emphysema, and intrapulmonary shunting [1,2]. Mechanical ventilation also causes secondary lung injury such as focal emphysema, pulmonary edema, and fibrosis [1,3]. Meanwhile, small tidal volume ventilation can decrease lung damage caused by shearing forces and benefit ARDS patients in terms of mortality and ventilator-free days [4]. This strategy has become the standard lung-protective ventilation strategy in ARDS. Theoretically, pulmonary atelectasis is the major pathological change in ARDS, and an experimental study has shown that tidal ventilation 
at low airway pressures can augment lung injury [5]. Thus, lung recruitment may improve hypoxemia by opening the collapsed lung and decreasing the intrapulmonary shunt (ventilation/perfusion mismatch) $[1,6]$.

Recently, lung recruitment maneuvers (LRMs) have been shown to safely recruit lung volume and improve blood oxygenation [7-10]. However, the recruitment cannot sustain the improvement of the clinical outcome of ARDS, and the application of high airway pressure could over-distend the aerated alveoli, leading to secondary ventilator-induced lung injury [11,12]. A stepwise LRM with incremental positive end expiratory pressure (PEEP) and a stable driving pressure can recruit most of the collapsed lung while minimizing hemodynamic compromise and inflammation [8,13]. However, after recruitment, a higher PEEP level could affect the sustainability of this effect [8,14]. Here, we created a modified stepwise LRM with decremental PEEP combined with lung-protective ventilation for patients with early ARDS. We hypothesized that a modified stepwise LRM with individualized moderate to high PEEP would result in a better clinical outcome compared with an established low-tidal-volume strategy in patients with early ARDS.

\section{Materials and Methods}

This prospective, randomized, controlled study was conducted in four intensive care units (ICUs) of Chi Mei Medical Center, Liouying, Taiwan. Patients were recruited from March 2009 through February 2012. The Institutional Review Board of the hospital approved the trial (IRB No.: CLH-0077), and legal substitute decision makers for each patient provided written informed consent. Trial registration: ClinicalTrials.gov, Identifier: NCT01114009.

\subsection{Patient Population}

Patients admitted to the ICUs who met the criteria for ARDS using the American-European Consensus Conference definition [2], who were on mechanical ventilation for $<72 \mathrm{~h}$, and who required a ratio of arterial oxygen tension to inspired oxygen fraction $(\mathrm{PaO} 2 / \mathrm{FiO} 2)$ of $\leq 250$ during invasive mechanical ventilation were enrolled. Before enrollment, oxygen saturation (SpO2) was established to $>88 \%$ and tidal volume (VT) to $6-8 \mathrm{~mL} / \mathrm{kg}$. PEEP and FiO2 were adjusted based on a standard PEEP protocol $[8,15,16]$. Arterial blood gas was measured after $30 \mathrm{~min}$ on these settings to confirm $\mathrm{PaO} 2 / \mathrm{FiO} 2$.

After enrollment, the participants were randomized to the experimental group (stepwise LRMs with lung-protective ventilation) or the control group (lung-protective ventilation only). The randomization sequence was created using SPSS for Windows (version 19.0, Chicago, IL, USA) with a 1:1 allocation using random block sizes of 2 and 4 by an independent doctor. The exclusion criteria were age $<18$ years, pregnancy, pneumothorax or subcutaneous emphysema or severe chronic respiratory lung disease, long-term ventilator dependency, acute brain injury or intracranial hypertension, neuromuscular disease, acute coronary syndrome or persistent ventricular tachyarrhythmias, and premorbid conditions with an expected 6-month mortality risk exceeding $50 \%$.

\subsection{Study Protocol}

The baseline mechanical ventilation was pressure control ventilation with VT of $\leq 6-8 \mathrm{~mL} / \mathrm{kg}$ of ideal body weight and a maintained plateau pressure of $\leq 30 \mathrm{cmH}_{2} \mathrm{O}$ (Table S1). For the experimental group, patients were sedated before LRMs and a neuromuscular-blocking agent was administered during LRMs. After ensuring hemodynamic stability, LRMs were performed on pressure control mode and at a peak airway pressure of $35 \mathrm{cmH}_{2} \mathrm{O}, \mathrm{PEEP}$ of $20 \mathrm{cmH}_{2} \mathrm{O}, \mathrm{FiO} 2$ of 1.0, respiratory rate of 15 breaths / $\mathrm{min}(\mathrm{bpm})$, and I/E ratio of 1:1. PEEP was increased by $3 \mathrm{cmH}_{2} \mathrm{O}$ every three breaths up to a peak airway pressure of $50 \mathrm{cmH}_{2} \mathrm{O}$, and the ventilation was kept for $2 \mathrm{~min}$. Thereafter, to find a derecruitment point, dynamic compliance (Cdyn) was observed with each breath, as displayed on the SERVO-i ventilator (MAQUET, Rastatt, Germany). We lowered the PEEP to $25 \mathrm{cmH}_{2} \mathrm{O}$, then decreased at $1 \mathrm{cmH}_{2} \mathrm{O}$ every three breaths until maximum Cdyn (derecruitment point, or call closing pressure) 
was identified. Once the maximum compliance PEEP was identified, PEEP was increased to $35 \mathrm{cmH}_{2} \mathrm{O}$ to reopen the lung for $2 \mathrm{~min}$, then returned to $2 \mathrm{cmH}_{2} \mathrm{O}$ above the derecruitment point (Figure 1). If the derecruitment point was not found, PEEP was set based on the original ARDSnet protocol [4]. We adjusted the $\mathrm{FiO} 2$ and respiratory rate to maintain adequate $\mathrm{SpO} 2$ and minute ventilation, and followed up arterial blood gas $30 \mathrm{~min}$ after. LRMs were performed every $8 \mathrm{~h}$ until maintaining $\mathrm{FiO} 2$ $\leq 0.40$ and PEEP $\leq 10$ for $8 \mathrm{~h}$ or more. An additional recruitment maneuver was performed following each disconnect from the ventilator. If $\mathrm{PaO} 2 / \mathrm{FiO} 2$ levels were stable or increasing for $12 \mathrm{~h}$ or more after LRMs, titrating of PEEP was started with decreases of $2 \mathrm{cmH}_{2} \mathrm{O}$ every $8 \mathrm{~h}$.

\section{Lung recruitment maneuver protocol}

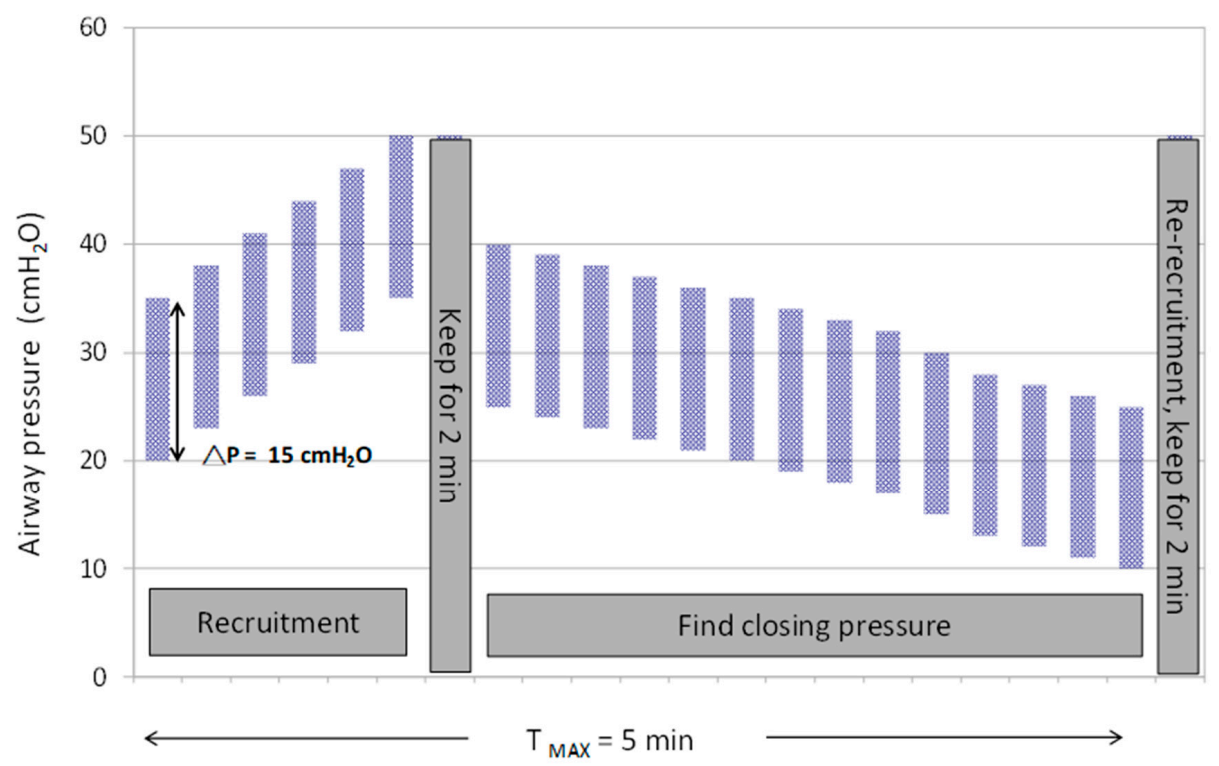

Figure 1. Diagram of a stepwise lung recruitment maneuver followed by decremental positive end-expiratory pressure titration applied in the study. ( $\mathrm{T}_{\mathrm{MAX}}$, maximum time).

If hypotension (systolic blood pressure $<80 \mathrm{mmHg}$ ) or bradycardia ( $<60 \mathrm{bpm}$ ) was observed, LRMs were immediately held and a fluid challenge or inotropic agent was administered to maintain haemodynamic stability. The mechanical ventilators used were SERVO-i with Open Lung Tool software, which enables real-time monitoring of respiratory system compliance. SERVO-i continuously displays PEEP, inspired and expired VT, and Cdyn. The graphical display of Cdyn indicates the response of the patient's respiratory system mechanics to each change in PEEP.

In both groups, the patients were assessed for weaning readiness for a spontaneous breathing trial daily when $\mathrm{FiO} 2 \leq 0.4$ and $\mathrm{PEEP} \leq 10$.

\subsection{Data Collection and Measurements}

Data on demographics, the Acute Physiology and Chronic Health Evaluation (APACHE) II score [17], the multiple-organ dysfunction score [18], and clinical conditions associated with ARDS were collected. We recorded the respiratory system mechanics, gas exchange, and hemodynamic data at baseline and at $8 \mathrm{~h}$ intervals thereafter until extubation or day 7 after enrollment. On each day, the lowest and highest values of each parameter were recorded. The patients were followed until day 60 or until discharge while breathing without assistance.

The primary outcomes were 28-day mortality, ventilator-free and ICU-free days at 28 days, and length of ICU and hospital stay after randomization. The secondary outcomes were ventilator-free and ICU-free days among survivors, 60-day mortality, incidence of barotrauma, and transient adverse events during LRMs. Additionally, we compared PEEP, FiO2, plateau pressure, VT, respiratory rate, Cdyn, and gas exchange between groups. 


\subsection{Statistical Analysis}

After the first block of 40 patients had been enrolled, a beneficial effect of the LRM approach on ventilator-free days became evident, and we performed an interim analysis. We estimated that a minimal sample of 120 patients was required, with an $\alpha$ of less than 0.05 and a $\beta$ of greater than $80 \%$. Continuous variables were expressed as mean \pm standard deviation with normal distribution or medians (interquartile range, IQR) with non-normal distribution. These variables were compared using the Wilcoxon rank-sum test or Student's independent $t$ test, as appropriate. Categorical variables were compared using the chi-square test or Fisher's exact test. A multivariate stepwise logistic regression model was used to identify risk factors for mortality.

All analyses followed the intention-to-treat principle, considering all patients in the treatment groups to which they were randomly assigned. In a planned secondary analysis of 28-day mortality, ICU mortality, and hospital mortality, we adjusted for age, APACHE II score, and sepsis. The difference in 60-day mortality between groups was compared using the Kaplan-Meier survival analysis with the log-rank test. All statistical analyses were conducted using SPSS for Windows (version 19.0, Chicago, IL, USA), and $p<0.05$ was considered statistically significant.

\section{Results}

\subsection{Patients}

We screened 254 patients with ARDS; 134 were excluded for reasons listed in Figure 2. The remaining 120 patients were equally randomized to the LRM and control groups. The baseline characteristics were similar between the two groups (Table 1). The most common causes of lung injury were pneumonia $(81.7 \%)$, non-pulmonary sepsis $(10.0 \%)$, and acute pancreatitis $(3.3 \%)$. All patients received sedatives prior to the recruitment maneuvers and 52 patients $(86.7 \%)$ received neuromuscular-blocking agents in LRM groups compared with 24 patients $(40 \%)$ in the control group $(p<0.001)$. None had received prone position in either group of patients. Echocardiography was performed in 98 patients $(81.7 \%)$ and 10 patients $(8.3 \%)$ had impaired left ventricular contractility, 16 patients $(13.3 \%)$ had mild to moderate pulmonary hypertension. All patients in the LRM group received at least one recruitment maneuver following the initial recruitment maneuver. The median times of LRMs performed were 5 times (IQR, $4-9$ times).

Table 1. Baseline characteristics of study subjects.

\begin{tabular}{|c|c|c|c|}
\hline Characteristics & LRM Group $(n=60)$ & Control Group $(n=60)$ & $p$ Value \\
\hline Age, mean (SD) & $66.8(16.1)$ & $63.7(20.8)$ & 0.37 \\
\hline Female sex & $15(25.0)$ & $16(26.7)$ & 0.84 \\
\hline APACHE II score, mean (SD) & $20.4(5.8)$ & $21.5(6.0)$ & 0.33 \\
\hline MODS, mean (SD) & $8.3(2.5)$ & $9.0(2.7)$ & 0.18 \\
\hline $\mathrm{PaO}_{2} / \mathrm{FiO}_{2}$, mean (SD) & $133.4(47.0)$ & $129.7(42.0)$ & 0.66 \\
\hline $\begin{array}{l}\text { Tidal volume, } \mathrm{mL} / \mathrm{kg} \text { of ideal body weight, } \\
\text { mean (SD) }\end{array}$ & $8.7(2.1)$ & $8.4(1.8)$ & 0.37 \\
\hline Minute ventilation, mean (SD) & $11.0(3.9)$ & $10.9(3.4)$ & 0.86 \\
\hline Total respiratory rate, mean (SD) & $22(6)$ & $23(7)$ & 0.36 \\
\hline $\begin{array}{l}\text { Cause of lung injury } \\
\text { Pneumonia }(\%)\end{array}$ & $52(86.7)$ & $46(76.7)$ & \\
\hline Non-pulmonary sepsis (\%) & $4(6.7)$ & $8(13.3)$ & \\
\hline Multiple transfusion (\%) & $0(0)$ & $2(3.3)$ & \\
\hline Acute pancreatitis (\%) & $2(3.3)$ & $2(3.3)$ & \\
\hline Others (\%) & $2(3.3)$ & $2(3.3)$ & \\
\hline
\end{tabular}

SD standard deviation, APACHE Acute Physiology and Chronic Health Evaluation, MODS multiple organ dysfunction syndrome, $\mathrm{PaO}_{2}$ partial pressure of arterial oxygen, $\mathrm{FiO}_{2}$ fraction of inspired oxygen. 


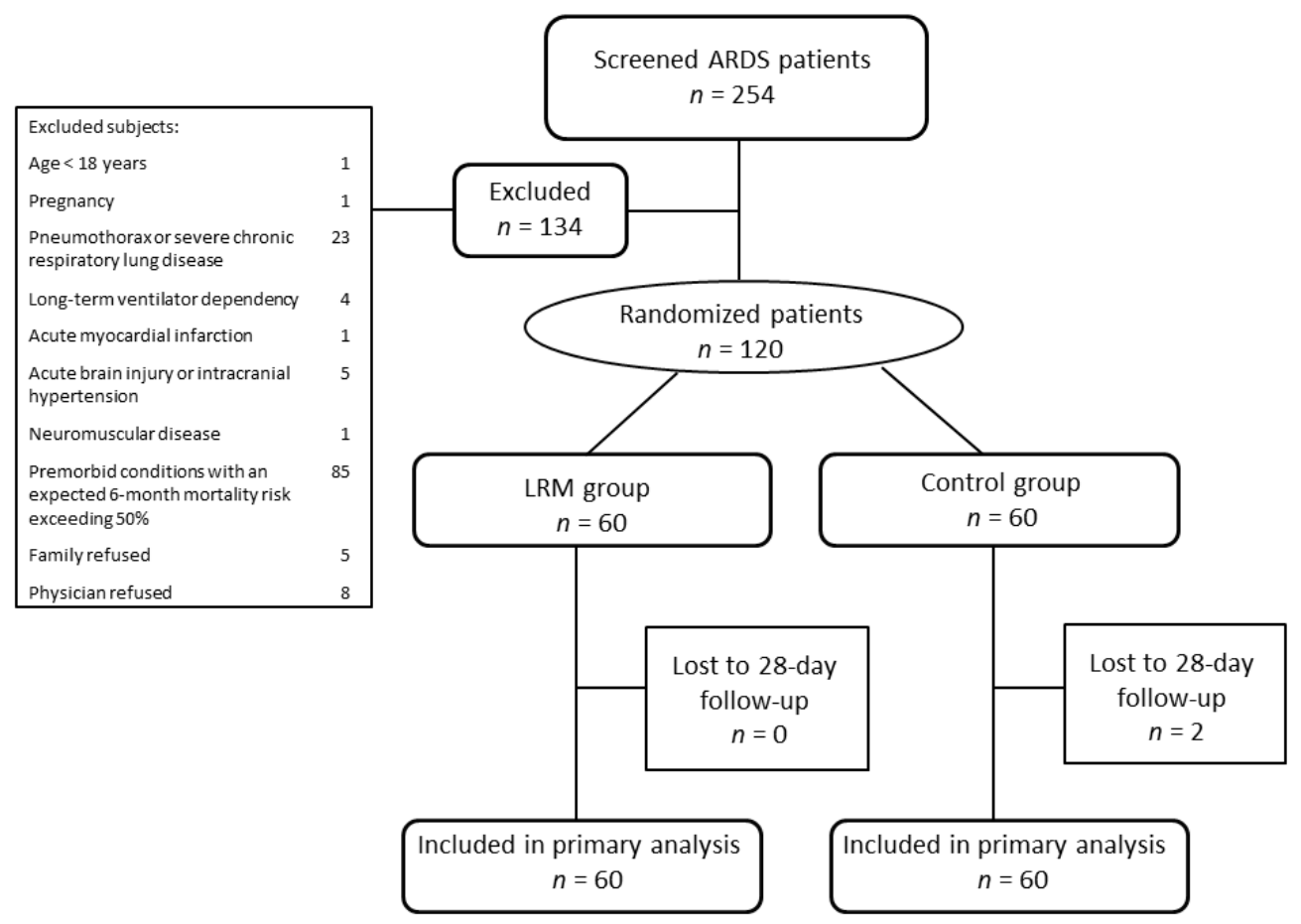

Figure 2. Flow chart of the study. ARDS, acute respiratory distress syndrome, LRM, lung recruitment maneuver.

\subsection{Primary and Secondary Outcome Data}

Two patients were lost to follow up in the control group. No significant difference in 28-day mortality, ICU mortality, and hospital mortality existed between the LRM and control groups (28\% vs. $30 \%, p=0.84,33.3 \%$ vs. $33.3 \%, p=1.0,35.0 \%$ vs. $38.3 \%, p=0.71$, respectively) (Table 2 ). The Kaplan-Meier survival curve demonstrates that there was no statistically significant difference in 60-day all-cause mortality between the groups ( $p=0.47$, log-rank test) (Figure 3).

Table 2. Primary and secondary outcomes.

\begin{tabular}{|c|c|c|c|c|}
\hline \multirow{2}{*}{ Outcomes } & LRM Group & Control Group & \multirow{2}{*}{$\begin{array}{l}\text { Relative Risk (95\% } \\
\text { Confidence Interval) }\end{array}$} & \multirow{2}{*}{$p$ Value } \\
\hline & $(n=60)$ & $(n=60)$ & & \\
\hline \multicolumn{5}{|l|}{ Death } \\
\hline Death during the first 28 day & $17(28.3 \%)$ & $18(30.0 \%)$ & $0.98(0.78-1.23)$ & 0.84 \\
\hline ICU mortality & $20(33.3 \%)$ & $20(33.3 \%)$ & $1.00(0.78-1.29)$ & 1.0 \\
\hline Hospital mortality & $21(35.0 \%)$ & $23(38.3 \%)$ & $0.95(0.72-1.25)$ & 0.71 \\
\hline Barotrauma & $2(3.3 \%)$ & $4(6.7 \%)$ & $0.97(0.89-1.05)$ & 0.40 \\
\hline \multicolumn{5}{|l|}{ Ventilator-free days ${ }^{a, b}$} \\
\hline Overall population & $11(0-20)$ & $4(0-16)$ & & 0.16 \\
\hline Patients with 28-day survival & $18(0-21)$ & $13(1-18)$ & & 0.04 \\
\hline \multicolumn{5}{|l|}{ ICU-free days ${ }^{a, b}$} \\
\hline Overall population & $7(0-19)$ & $0(0-15)$ & & 0.10 \\
\hline Patients with 28-day survival & $16(0-20)$ & $11(0-16)$ & & 0.03 \\
\hline $\begin{array}{l}\text { Days of mechanical ventilation } \\
\text { a }\end{array}$ & $11(6-24)$ & $12(7-23)$ & & 0.60 \\
\hline Days of intensive care & $12(7-24)$ & $14(7-27)$ & & 0.54 \\
\hline Days of hospitalization $^{\text {a }}$ & $22(13-34)$ & $23(10-38)$ & & 0.62 \\
\hline
\end{tabular}

LRM lung recruitment maneuver, ICU intensive care unit. a Continuous data are presented as median (interquartile range). ${ }^{\mathrm{b}}$ Two patients lost to follow-up were excluded for analysis. 

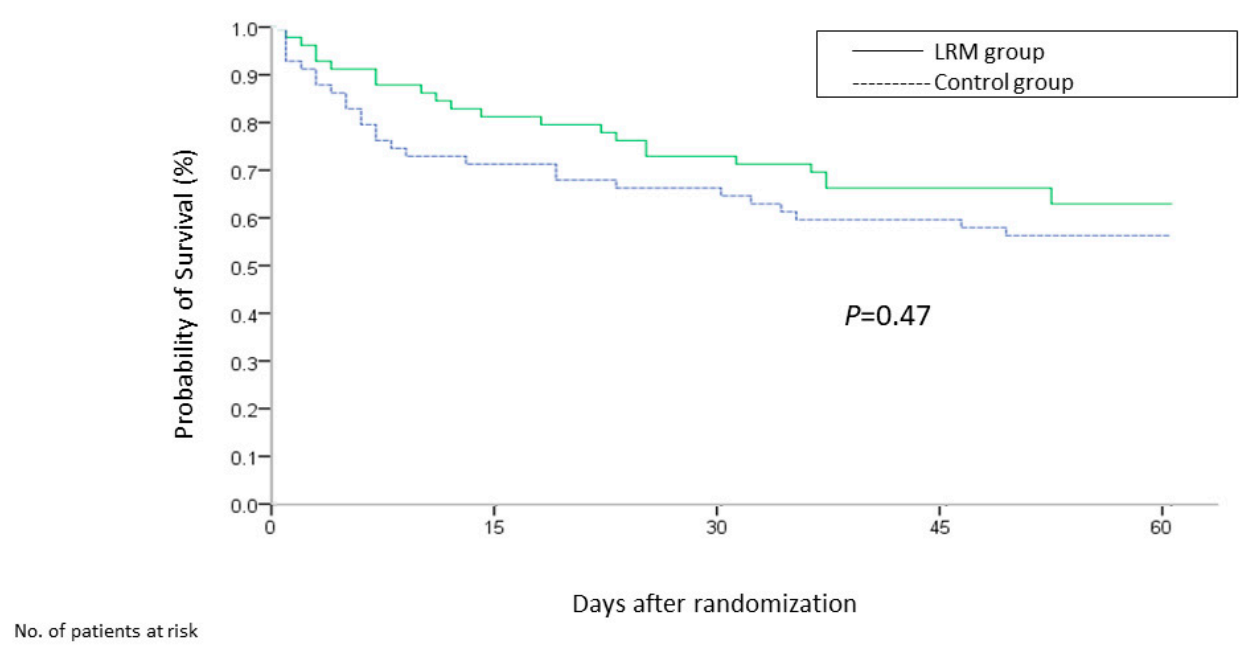

Days after randomization

\begin{tabular}{|c|c|c|c|c|c|}
\hline LRM group & 60 & 49 & 43 & 39 & 37 \\
\hline Control group & 60 & 43 & 39 & 35 & 33 \\
\hline
\end{tabular}

Figure 3. Probability of survival from day of randomization to day 60 among patients in the lung recruitment maneuver (LRM) group and control group.

\subsection{Mechanical Ventilation Days and Other Clinical Outcomes}

There were no significant differences in ventilator-free days and ICU-free days at 28 days in both groups (Table 2). On subgroup analysis, when we compared survivors at 28 days who were in LRM group with those in control group, we found that survivors in the LRM group had significantly higher ventilator-free days at 28 days (median $=18(\mathrm{IQR}, 0-21)$ vs. median $=13(\mathrm{IQR}, 1-18)$, respectively; $p=0.04)$ and significantly higher ICU-free days at 28 days (median $=16(\mathrm{IQR}, 0-20)$ vs. median $=11(\mathrm{IQR}$, $0-16)$, respectively; $p=0.03$ ). The median duration of hospitalization among survivors was 22 days (IQR, 15-34 days) in the LRM group and 34 days (IQR, 20-47 days) in the control group ( $p=0.07$ ).

\subsection{Respiratory and Hemodynamic Variables}

The mean VT and plateau pressures were similar in both groups on days 1,3, and 7 and within the target range (Table 3). Cdyn was significantly higher on days 1, 3, and 7 in the LRM group. The closing pressure after recruitment maneuver could be found in 16 patients in the LRM group. The arterial $\mathrm{pH}, \mathrm{PaO} 2$, and carbon dioxide were similar in both groups at all three times, but the $\mathrm{PaO} 2 / \mathrm{FiO} 2$ was significantly higher in the LRM group on days 1 and 3 . On day 1 , systolic and diastolic blood pressures were also significantly higher in the LRM group, while heart and respiratory rates were similar in both groups on days 1,3 , and 7 .

\subsection{Adverse Events}

Two LRM patients (3.3\%) vs. four controls $(6.7 \%)$ developed a barotrauma episode $(p=0.40)$. In the LRM group, 32 patients (53.3\%) experienced transient hypotension, $5(8.3 \%)$ had $\mathrm{SpO} 2$ of $<88 \%$, $6(10 \%)$ were associated with arrhythmia, and none was associated with cardiac arrest. 
Table 3. Respiratory and hemodynamic parameters.

\begin{tabular}{|c|c|c|c|c|c|c|c|c|c|}
\hline & & Day 1 & & & Day 3 & & & Day 7 & \\
\hline Variables & LRM Group & Control Group & $p$ Value & LRM Group & Control Group & $p$ Value & LRM Group & Control Group & $p$ Value \\
\hline No. of patients & 60 & 60 & & 57 & 55 & & 35 & 37 & \\
\hline $\begin{array}{l}\text { Tidal volume, mean (SD) }{ }^{\mathrm{a}} \\
\mathrm{mL} / \mathrm{kg} \text { of ideal body weight }\end{array}$ & $7.7(1.0)$ & $7.6(0.9)$ & 0.57 & $7.8(1.2)$ & $7.6(1.0)$ & 0.49 & $7.9(1.3)$ & $7.8(1.5)$ & 0.62 \\
\hline Total respiratory rate, mean (SD) ${ }^{a}$ & $21(4)$ & $22(5)$ & 0.11 & $20(4)$ & $21(5)$ & 0.14 & $20(5)$ & $22(6)$ & 0.11 \\
\hline Plateau pressure, mean (SD) ${ }^{a}$ & $25.3(3.9)$ & $25.7(4.6)$ & 0.73 & $24.0(4.7)$ & $26.1(4.3)$ & 0.07 & $25.2(5.7)$ & $26.2(4.4)$ & 0.74 \\
\hline $\mathrm{C}_{\mathrm{dyn}}, \operatorname{median}(\mathrm{IQR})^{\mathrm{a}}$ & $28.2(25.8-36.3)$ & $25.3(21.5-28.5)$ & 0.001 & $31.0(27.0-36.2)$ & $26.7(20.9-30.7)$ & 0.001 & $32(28.3-38.0)$ & $26.5(26.0-31.0)$ & 0.002 \\
\hline $\mathrm{FiO}_{2}$, median $(\mathrm{IQR})^{\mathrm{a}}$ & $0.50(0.47-0.62)$ & $0.55(0.47-0.72)$ & 0.37 & $0.38(0.35-0.46)$ & $0.45(0.40-0.55)$ & 0.005 & $0.35(0.30-0.40)$ & $0.40(0.35-0.49)$ & 0.04 \\
\hline $\mathrm{pH}$, mean $(\mathrm{SD})^{\mathrm{a}}$ & $7.40(0.07)$ & $7.34(0.33)$ & 0.71 & $7.43(0.06)$ & $7.43(0.06)$ & 0.42 & $7.46(0.07)$ & $7.46(0.06)$ & 0.74 \\
\hline $\mathrm{PaCO}_{2}$, mean $(\mathrm{SD})^{\mathrm{a}}$ & $35.7(6.1)$ & $36.1(9.2)$ & 0.70 & $35.8(6.3)$ & $37.0(5.6)$ & 0.23 & $37.3(6.7)$ & $36.5(6.5)$ & 0.85 \\
\hline $\mathrm{PaO}_{2}$, mean (SD) ${ }^{\mathrm{a}}$ & $92.1(28.2)$ & $84.0(28.8)$ & 0.06 & $82.9(13.9)$ & $79.3(12.3)$ & 0.19 & 80.6 (16.5) & $80.3(22.5)$ & 0.40 \\
\hline $\mathrm{PaO}_{2} / \mathrm{FiO}_{2}$, mean (SD) ${ }^{\mathrm{a}}$ & $174.8(67.8)$ & $150.8(62.8)$ & 0.047 & $209.7(72.7)$ & $174.1(55.1)$ & 0.004 & $222.7(69.7)$ & $200.3(71.3)$ & 0.21 \\
\hline Heart rate, mean (SD) & $98(21)$ & $99(20)$ & 0.65 & 89 (17) & $93(18)$ & 0.14 & $94(20)$ & $96(17)$ & 0.47 \\
\hline Systemic blood pressure, mean (SD) & $133(20)$ & $119(20)$ & $<0.001$ & $136(17)$ & $130(23)$ & 0.09 & $137.7(22.6)$ & $136.8(28)$ & 0.92 \\
\hline Diastolic blood pressure, mean (SD) & $70(12)$ & $65(11)$ & 0.03 & $72(11)$ & $69(12)$ & 0.10 & $74.6(18.3)$ & $73.0(14.7)$ & 0.88 \\
\hline
\end{tabular}

SD standard deviation, IQR interquartile range, $\mathrm{C}_{\text {dyn }}$ dynamic compliance, $\mathrm{FiO}_{2}$ fraction of inspired oxygen, $\mathrm{PEEP}$ positive end-expiratory pressure, PaO $\mathrm{O}_{2}$ partial pressure of arterial oxygen, $\mathrm{PaCO}_{2}$ partial pressure of arterial carbon dioxide; ${ }^{\mathrm{a}}$ Data shown were derived from the average value obtained for each patient over 3 measurements each day; values were recorded on days 1,3 , and 7 after enrolment. 


\section{Discussion}

This study has several significant findings. To our knowledge, this is the first randomized, controlled trial demonstrating that early application of a stepwise LRM combined with lung-protective ventilation improved patient outcomes, with increased ventilator-free and ICU-free days among survivors. Although no significant difference in 28-day mortality existed between groups, LRMs may have some clinical benefits if applied in the very early phase of ARDS.

Recruitment maneuvers are categorized based on the intervention method. A stepwise LRM with incremental PEEP and a stable driving pressure can achieve most attainable recruitment while minimizing hemodynamic compromise and inflammation [19]. However, this strategy is usually time-consuming and not clinically practical, and the inflation time of LRMs is thought to have less importance in determining recruiting success [20]. Lim et al. performed an extended sigh technique in 20 patients, and an 8 min average was needed [21]. Borges et al. used a stepwise maximum-recruitment strategy that took $20 \mathrm{~min}$ for each recruitment maneuver performed [8]. Here, we created a modified stepwise LRM combining these two methods, and only 4-5 min were needed for each maneuver; it was thus less time-consuming. Besides, according to previous studies using a decremental PEEP trial to identify an optimal post-LRM PEEP level that can significantly maintain oxygenation benefits for at least 4-6 h, we added the individualized PEEP levels after LRMs [8,22]. Our modified stepwise LRM protocol is similar to that of Kacmarek et al., which used stepwise incremental PEEP, a stable driving pressure, and post-recruiting decremental PEEP [23]. However, our recruiting peak airway pressure was lower, at $50 \mathrm{cmH}_{2} \mathrm{O}$, to prevent over-distension related to hemodynamic adverse events and pneumothorax. Furthermore, the rate of barotrauma (3\% vs. $8 \%$ ), desaturation (8.3\% vs. 34\%), and arrhythmia (10\% vs. 15\%) was lower in our LRM group than in Kacmarek et al.'s LRM group [23].

LRMs are crucial in ARDS; however, their impact on the clinical outcomes of ARDS is variable and uncertain. One possible influencing factor of the effectiveness of LRMs is the initial application time. Cavalcanti et al. performed a large multicenter, randomized trial (the Alveolar Recruitment for ARDS Trial, ART) and their findings do not support the routine use of lung recruitment maneuver and PEEP titration in patients with moderate to severe ARDS [24]. For more than 22\% patients in that study, however, the duration of ARDS at randomization is above $36 \mathrm{~h}$. In contrast, most of our LRM patients (82\%) started LRMs within $24 \mathrm{~h}$ after ARDS was diagnosed. Further analysis of our LRM group revealed that patients who received LRMs in very early ARDS ( $<24 \mathrm{~h}$ from ARDS onset) had a significantly lower 28-day mortality, ICU mortality, and hospital mortality, compared with patients who received LRMs within 25-72 h after ARDS onset. Like the beneficial recruiting effect of early prone positioning in ARDS patients, LRMs may better recruit the collapsed alveoli in the early exudative hyaline membrane phase of ARDS than in the late fibroproliferative phase $[25,26]$. Clinically, Grasso et al. has shown that LRMs successfully improved the oxygenation only in patients with early ARDS who were on the ventilator for $<48 \mathrm{~h}$ and without impaired chest wall mechanics [26]. Borges et al. used quantitative computed tomography to document that LRMs can reduce alveolar collapse in most patients with early ARDS [8]. Besides, in order to perform LRMs, more patients $(86.7 \%)$ in our study received neuromuscular-blocking agents in LRM groups compared with patients $(40 \%)$ in the control group. Papazian et al. found that treatment with the neuromuscular blocking agent in early severe ARDS increased the numbers of ventilator-free days and days outside the ICU [27]. Therefore, LRMs may improve the clinical outcome if applied in very early ARDS.

The etiology of ARDS is another influencing factor of the effectiveness of LRMs. Extra-pulmonary ARDS is believed to be more recruitable [25,28], which can be explained by the higher potential for recruitment in alveolar collapse than in consolidation. Unlike the randomized, controlled trial by Kacmarek et al. and Meade et al., which comprised nearly $50 \%$ of patients with extra-pulmonary ARDS, almost $90 \%$ of our enrolled patients had pulmonary ARDS in both the LRM and control groups [23,29]. Like two previous studies, our study showed that the LRM group had no significant survival benefit compared with the control group. However, in ARDS survivors, our data showed that LRMs indeed lengthened the ventilator-free and ICU-free days, which could be explained by using LRMs in early 
ARDS. In pulmonary ARDS, acute lung damage induces interstitial and alveolar edema, followed by rapid fibroblastic cell proliferation and fibrosis [30]. If we perform LRMs in patients with pulmonary ARDS in the very early stage before their pulmonary fibroblastic cell activation, the recruitment effect may become better. However, if the pulmonary damage is so severe that alveolar space is altered by hemorrhage and purulent exudate, the recruitment effect will diminish. Thus, the beneficial effect could be seen in our ARDS survivors. This hypothesis can be proved by Lamy et al.'s morphological study, which showed that PEEP is responsive to patients with less severe lung damage with diffuse congestion and microatelectasis, but this response disappears in late ARDS [31]. Negri et al. also reported an increased collagen content in pulmonary ARDS compared with extra-pulmonary ARDS in the early phase [32]. Therefore, LRMs applied in early ARDS may be more helpful in pulmonary ARDS.

The most common adverse events when performing LRMs are vital sign abnormalities such as hypotension, desaturation, and bradycardia. These hemodynamic adverse events are transient and self-limited, but a small proportion of patients (1\%) still need an early cessation of LRMs until they are hemodynamically stable [12]. However, in our study, there was no incidence of LRM termination due to adverse events. There was also low incidence of LRMs associated with pneumothorax, consistent with other previous studies $[12,23,33]$.

Our study has some limitations. First, it was difficult to set the "ideal optimal PEEP" to get the maximum compliance on the deflation limb of the pressure-volume curve of the LRM patients. Optimal PEEP after recruitment could only be found in $25 \%$ patients in our study, which may be attributed to the majority of patients who had pulmonary ARDS. The heterogeneous distribution of consolidated/collapsed lung results in a wide variation in pulmonary compliance, making it hard to find the optimal PEEP. Second, our trial was performed in a single institute and the number of cases was limited. Third, only $40 \%$ patients received chest CT scan, and the homogeneous of two groups for lung morphological aspects could not be evaluated. Lastly, our primary outcome revealed no difference between the LRM and control groups, and only subgroup analysis showed significant clinical benefits of LRMs. This beneficial impact could have been more notable if our number of cases was larger.

\section{Conclusions}

Applying stepwise LRMs combined with lung-protective ventilation in early ARDS can improve patient outcomes, increasing ventilator-free and ICU-free days among 28-day survivors.

Supplementary Materials: The following are available online at http:/ /www.mdpi.com/2077-0383/8/2/231/s1, Table S1: Summary of the ventilator procedure.

Author Contributions: Conceptualization, S.-C.K., W.-L.C. and W.-L.L.; Data curation, S.-C.K., W.-L.C., and H.-C.C.; Formal analysis, Y.-L.H., W.-L.C., and W.-L.L.; Investigation, S.-C.K., W.-L.C., C.-M.W., H.-C.C., and W.-L.L.; Methodology, S.-C.K. and W.-L.L.; Supervision, W.-L.L.; Validation, Y.-L.H., C.-M.W., and W.-L.L.; Writing—original draft, S.-C.K., Y.-L.H., and W.-L.L.; Writing—review and editing, S.-C.K., Y.-L.H., W.-L.C., C.-M.W., and W.-L.L.

Funding: This research was funded by Chi Mei Medical Center, Liouying, grant number: CLFHR0991423.

Acknowledgments: The authors would like to thank all respiratory therapists and nursing teams for the excellent care provided to the patients during the study.

Conflicts of Interest: The authors declare no conflict of interest.

\section{References}

1. Lachmann, B. Open up the lung and keep the lung open. Intensive Care Med. 1992, 18, 319-321. [CrossRef] [PubMed]

2. Bernard, G.R.; Artigas, A.; Brigham, K.L.; Carlet, J.; Falke, K.; Hudson, L.; Lamy, M.; Legall, J.R.; Morris, A.; Spragg, R. The American-European Consensus Conference on ARDS. Definitions, mechanisms, relevant outcomes, and clinical trial coordination. Am. J. Respi.r Crit. Care Med. 1994, 149, 818-824. [CrossRef] [PubMed] 
3. Slutsky, A.S. Lung injury caused by mechanical ventilation. Chest. 1999, 116, 9s-15s. [CrossRef] [PubMed]

4. The Acute Respiratory Distress Syndrome Network; Brower, R.G.; Matthay, M.A.; Morris, A.; Schoenfeld, D.; Thompso, B.T.; Wheeler, A. Ventilation with lower tidal volumes as compared with traditional tidal volumes for acute lung injury and the acute respiratory distress syndrome. N. Engl. J. Med. 2000, 342, 1301-1308.

5. Muscedere, J.G.; Mullen, J.B.; Gan, K.; Slutsky, A.S. Tidal ventilation at low airway pressures can augment lung injury. Am. J. Respir. Crit. Care Med. 1994, 149, 1327-1334. [CrossRef] [PubMed]

6. Pelosi, P.; Cadringher, P.; Bottino, N.; Panigada, M.; Carrieri, F.; Riva, E.; Lissoni, A.; Gattinoni, L. Sigh in acute respiratory distress syndrome. Am. J. Respir. Crit. Care Med. 1999, 159, 872-880. [CrossRef] [PubMed]

7. Cereda, M.; Emami, K.; Kadlecek, S.; Xin, Y.; Mongkolwisetwara, P.; Profka, H.; Barulic, A.; Pickup, S.; Mansson, S.; Wollmer, P.; et al. Quantitative imaging of alveolar recruitment with hyperpolarized gas MRI during mechanical ventilation. J. Appl. Physiol. 2011, 110, 499-511. [CrossRef]

8. $\quad$ Borges, J.B.; Okamoto, V.N.; Matos, G.F.; Caramez, M.P.; Arantes, P.R.; Barros, F.; Souza, C.E.; Victorino, J.A.; Kacmarek, R.M.; Barbas, C.S.; et al. Reversibility of lung collapse and hypoxemia in early acute respiratory distress syndrome. Am. J. Respir. Crit. Care Med. 2006, 174, 268-278. [CrossRef]

9. Albaiceta, G.M.; Luyando, L.H.; Parra, D.; Menendez, R.; Calvo, J.; Pedreira, P.R.; Taboada, F. Inspiratory vs. expiratory pressure-volume curves to set end-expiratory pressure in acute lung injury. Intensive Care Med. 2005, 31, 1370-1378. [CrossRef]

10. Medoff, B.D.; Harris, R.S.; Kesselman, H.; Venegas, J.; Amato, M.B.; Hess, D. Use of recruitment maneuvers and high-positive end-expiratory pressure in a patient with acute respiratory distress syndrome. Crit. Care Med. 2000, 28, 1210-1216. [CrossRef]

11. Hodgson, C.; Keating, J.L.; Holland, A.E.; Davies, A.R.; Smirneos, L.; Bradley, S.J.; Tuxen, D. Recruitment manoeuvres for adults with acute lung injury receiving mechanical ventilation. Cochrane Database Syst. Rev. 2009. [CrossRef]

12. Fan, E.; Wilcox, M.E.; Brower, R.G.; Stewart, T.E.; Mehta, S.; Lapinsky, S.E.; Meade, M.O.; Ferguson, N.D. Recruitment maneuvers for acute lung injury: A systematic review. Am. J. Respir. Crit. Care Med 2008, 178, 1156-1163. [CrossRef] [PubMed]

13. Silva, P.L.; Moraes, L.; Santos, R.S.; Samary, C.; Ramos, M.B.; Santos, C.L.; Morales, M.M.; Capelozzi, V.L.; Garcia, C.S.; de Abreu, M.G.; et al. Recruitment maneuvers modulate epithelial and endothelial cell response according to acute lung injury etiology. Crit. Care Med. 2013, 41, e256-e265. [CrossRef] [PubMed]

14. Gattinoni, L.; Caironi, P.; Cressoni, M.; Chiumello, D.; Ranieri, V.M.; Quintel, M.; Russo, S.; Patroniti, N.; Cornejo, R.; Bugedo, G. Lung recruitment in patients with the acute respiratory distress syndrome. N. Engl. J. Med. 2006, 354, 1775-1786. [CrossRef] [PubMed]

15. Ferguson, N.D.; Kacmarek, R.M.; Chiche, J.D.; Singh, J.M.; Hallett, D.C.; Mehta, S.; Stewart, T.E. Screening of ARDS patients using standardized ventilator settings: Influence on enrollment in a clinical trial. Intensive Care Med. 2004, 30, 1111-1116. [CrossRef]

16. Villar, J.; Perez-Mendez, L.; Blanco, J.; Anon, J.M.; Blanch, L.; Belda, J.; Santos-Bouza, A.; Fernández, R.L.; Kacmarek, R.M.; Spanish Initiative for Epidemiology, Stratification, and Therapies for ARDS (SIESTA) Network. A universal definition of ARDS: The $\mathrm{PaO} 2 / \mathrm{FiO} 2$ ratio under a standard ventilatory setting - A prospective, multicenter validation study. Intensive Care Med. 2013, 39, 583-592. [CrossRef]

17. Knaus, W.A.; Draper, E.A.; Wagner, D.P.; Zimmerman, J.E. APACHE II: A severity of disease classification system. Crit. Care Med. 1985, 13, 818-829. [CrossRef]

18. Marshall, J.C.; Cook, D.J.; Christou, N.V.; Bernard, G.R.; Sprung, C.L.; Sibbald, W.J. Multiple organ dysfunction score: A reliable descriptor of a complex clinical outcome. Crit. Care Med. 1995, 23, 1638-1652. [CrossRef]

19. Keenan, J.C.; Formenti, P.; Marini, J.J. Lung recruitment in acute respiratory distress syndrome: What is the best strategy? Curr. Opin. Crit. Care. 2014, 20, 63-68. [CrossRef]

20. Marini, J.J. Recruitment maneuvers to achieve an "open lung"-whether and how? Crit. Care Med. 2001, 29, 1647-1648. [CrossRef]

21. Lim, C.M.; Koh, Y.; Park, W.; Chin, J.Y.; Shim, T.S.; Lee, S.D.; Kim, W.S.; Kim, D.S.; Kim, W.D. Mechanistic scheme and effect of "extended sigh" as a recruitment maneuver in patients with acute respiratory distress syndrome: A preliminary study. Crit. Care Med. 2001, 29, 1255-1260. [CrossRef] [PubMed]

22. Girgis, K.; Hamed, H.; Khater, Y.; Kacmarek, R.M. A decremental PEEP trial identifies the PEEP level that maintains oxygenation after lung recruitment. Respir. Care. 2006, 51, 1132-1139. [PubMed] 
23. Kacmarek, R.M.; Villar, J.; Sulemanji, D.; Montiel, R.; Ferrando, C.; Blanco, J.; Koh, Y.; Soler, J.A.; Martínez, D.; Hernández, M.; et al. Open Lung Approach for the Acute Respiratory Distress Syndrome: A Pilot, Randomized Controlled Trial. Crit. Care Med. 2016, 44, 32-42. [CrossRef]

24. Writing Group for the Alveolar Recruitment for Acute Respiratory Distress Syndrome Trial (ART) Investigators; Cavalcanti, A.B.; Suzumura, É.A.; Laranjeira, L.N.; Paisani, D.M.; Damiani, L.P.; Guimarães, H.P.; Romano, E.R.; Regenga, M.M.; Taniguchi, L.N.T.; et al. Effect of lung recruitment and titrated positive end-expiratory pressure (peep) vs low peep on mortality in patients with acute respiratory distress syndrome: A randomized clinical trial. JAMA 2017, 318, 1335-1345.

25. Guerin, C.; Reignier, J.; Richard, J.C.; Beuret, P.; Gacouin, A.; Boulain, T.; Mercier, E.; Badet, M.; Mercat, A.; Baudin, O.; et al. Prone positioning in severe acute respiratory distress syndrome. N. Engl. J. Med. 2013, 368, 2159-2168. [CrossRef] [PubMed]

26. Grasso, S.; Mascia, L.; Del Turco, M.; Malacarne, P.; Giunta, F.; Brochard, L.; Slutsky, A.S.; Marco Ranieri, V. Effects of recruiting maneuvers in patients with acute respiratory distress syndrome ventilated with protective ventilatory strategy. Anesthesiology 2002, 96, 795-802. [CrossRef] [PubMed]

27. Papazian, L.; Forel, J.M.; Gacouin, A.; Penot-Ragon, C.; Perrin, G.; Loundou, A.; Jaber, S.; Arnal, J.M.; Perez, D.; Seghboyan, J.M.; et al. Neuromuscular blockers in early acute respiratory distress syndrome. N. Engl. J. Med. 2010, 363, 1107-1116. [CrossRef]

28. Foti, G.; Cereda, M.; Sparacino, M.E.; De Marchi, L.; Villa, F.; Pesenti, A. Effects of periodic lung recruitment maneuvers on gas exchange and respiratory mechanics in mechanically ventilated acute respiratory distress syndrome (ARDS) patients. Intensive Care Med. 2000, 26, 501-507. [CrossRef]

29. Meade, M.O.; Cook, D.J.; Guyatt, G.H.; Slutsky, A.S.; Arabi, Y.M.; Cooper, D.J.; Davies, A.R.; Hand, L.E.; Zhou, Q.; Thabane, L.; et al. Ventilation strategy using low tidal volumes, recruitment maneuvers, and high positive end-expiratory pressure for acute lung injury and acute respiratory distress syndrome: A randomized controlled trial. JAMA. 2008, 299, 637-645. [CrossRef]

30. Katzenstein, A.L.; Bloor, C.M.; Leibow, A.A. Diffuse alveolar damage-the role of oxygen, shock, and related factors. A review. Am. J. Pathol. 1976, 85, 209-228.

31. Lamy, M.; Fallat, R.J.; Koeniger, E.; Dietrich, H.P.; Ratliff, J.L.; Eberhart, R.C.; Tucker, H.J.; Hill, J.D. Pathologic features and mechanisms of hypoxemia in adult respiratory distress syndrome. Am. Rev. Respir. Dis. 1976, 114, 267-284. [PubMed]

32. Negri, E.M.; Hoelz, C.; Barbas, C.S.; Montes, G.S.; Saldiva, P.H.; Capelozzi, V.L. Acute remodeling of parenchyma in pulmonary and extrapulmonary ARDS. An autopsy study of collagen-elastic system fibers. Pathol. Res. Pract. 2002, 198, 355-361. [CrossRef] [PubMed]

33. Hodgson, C.; Goligher, E.C.; Young, M.E.; Keating, J.L.; Holland, A.E.; Romero, L.; Bradley, S.J.; Tuxen, D. Recruitment manoeuvres for adults with acute respiratory distress syndrome receiving mechanical ventilation. Cochrane Database Syst. Rev. 2016, 11, Cd006667. [CrossRef] [PubMed] 